\title{
Tensor Based Finite Element Model for the Calculation of Leakage Field in Magnetic Flux Leakage Testing
}

\author{
ALIMEY Fred John ${ }^{\text {a }}$, BAI Libing ${ }^{\text {a, } 1}$ and CHENG Yuhua ${ }^{\text {a }}$ \\ ${ }^{a}$ School of Automation Engineering, University of Electronic Science and \\ Technology of China, Chengdu, China
}

\begin{abstract}
Magnetic flux leakage (MFL) testing is a widely used electromagnetic nondestructive testing (ENDT) method, which has the ability to detect both surface and sub-surface defects in conductive materials. One of its best features is its ability to mathematically model field leakage from the defect area in a magnetized material. In this paper, we propose an optimized FEM model using geometrical weighted tensor (TBFEM), for the calculation of leakage field in MFL. This model using the Einstein's convention eliminates the bulky nature of traditional FEM based on its matrix algebra formation allowing for easy implementation and fast calculations. The proposed model achieves this by reducing the set of matrix equations into a single equation using suffixes which can then be solved with regular mathematical operations
\end{abstract}

Keywords. Finite element method (FEM), Einstein's convention, magnetic flux leakage, matrices, weighted tensors

\section{Introduction}

Over the years, electromagnetic nondestructive testing and evaluation (ENDT\&E) has become more and more popular with successful applications (in the aerospace, oil and gas, nuclear and transportation industries) for the determination of the quality and the structural health of components, machineries and equipment. At the core of most electromagnetic techniques that allow for numerical modelling is the finite element method (FEM). This is due to its flexibility and ability to model both linear and nonlinear boundary value problems (BVP). The main purpose of FEM is to determine the approximate solution of a boundary value problem (BVP), which is governed by a partial differential equation (PDE).

In this paper we propose a tensor based finite element model (TBFEM) for the calculation of leakage field in magnetic flux leakage (MFL) testing. MFL testing is a widely used electromagnetic nondestructive evaluation/testing (ENDT\&E) method that has the ability to detect both surface and sub-surface defects in ferromagnetic materials. The basic idea is to magnetize a material to saturation using a permanent magnet or yoke as shown in the Figure. 1 below. The presence of defect will cause varying magnetic field distribution in the material which in turn causes flux leakage. These changes in the material can then be detected using magnetic field sensors for further processing.

\footnotetext{
${ }^{1}$ Corresponding author, Bai Libing, School of Automation Engineering, University of electronic science and technology of China (UESTC); (E-mail: libing.bai@uestc.edu.cn).
} 


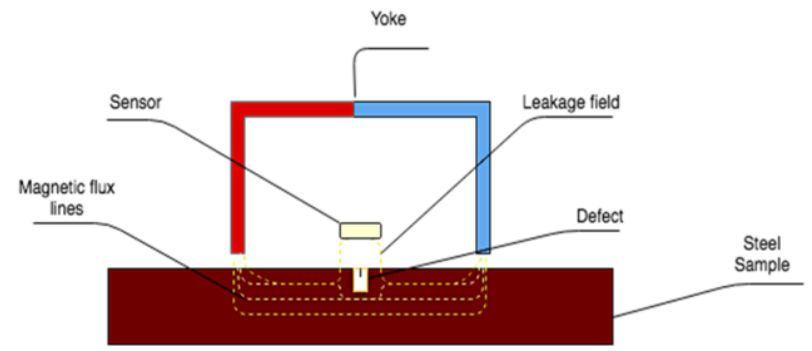

Figure. 1 Principle of magnetic flux leakage (MFL) testing

With MFL testing, two basic models are used for the prediction and visualization of magnetic field interaction with defect region. The analytical model, which was the first theoretical model to relate the shape of defects with the magnetic field strength, material permeability and magnetic field leakage [1], has contributed greatly to the understanding of how the MFL technique works. Even though this model greatly simplifies the difficulties associated with MFL analysis, too many assumptions are made, making it challenging for practical applications. More so, results from this model can only explain simple and regular defects [2]. The second basic model which is widely used is the numerical model. This model has shown more advantages as compared to the analytical model for MFL. According to Lord and Hwang [3], who first introduced the finite element method (FEM) to the calculation of the magnetic leakage field, proved through the study of how different shapes, angles, depth and width influence magnetic field. They then concluded that the only feasible solution for solving complex shape defects problems is through numerical calculation. Since then the finite element method (FEM) has been greatly explored and a lot has been achieved due to its flexibility and robustness. Even with all of its advantages, FEM has its challenges, some of which include excessive need for computing resources and time depending on the number of elements (matrixes) to be processed, as best results are greatly dependent on discretization (meshing) which makes the process time consuming and computationally expensive [4], [5]. In this paper, using the tensor based finite element model (TBFEM) which is derived from the Einstein's convention, we are able to calculate and simulate leakage field more efficiently, with a minimum computer specification, within a shorter time frame as compared to traditional FEM. The implementation is done in MATLAB RB2014 with a windows based computer of 1.4ghz speed, 4GB RAM.

\section{Magnetic leakage calculation using tensor based finite element model (TBFEM)}

\subsection{Magnetic leakage field calculation}

In MFL, calculating the magnetic leakage field means solving the Maxwell's equation under certain boundary conditions. Knowing that $\nabla \cdot B=0$ in static cases, $B$ can then be expressed in terms of magnetic vector potential $V$ for which $B=\nabla \times V$. Assuming $B=\mu \cdot H$ where $\mu$ is the permeability, then combining $B=\nabla \times V$ with $\nabla \times H=J$ where $H$ is the magnetic field intensity, and $J$ is the current density, the partial differential equation (PDE) in the equation (1) below can be obtained. 


$$
\nabla \times\left(\nabla \times\left(\frac{1}{\mu} \cdot B\right)\right)=J
$$

However, because $V$ is a vector potential, its boundary value problem (BVP) becomes difficult to solve. As shown in the introduction, the basic principle of MFL is to magnetize the sample using a permanent magnet or yoke, which means no conduction current is used within the domain, making $J=0$. For this reason, a magnetic scalar potential $V_{m}$ is used, so to describe the magnetic field for which the equations (2) - (4) below are satisfied within the domain.

$$
\begin{aligned}
& \nabla \times H=0 \\
& \nabla \times B=0 \\
& B \equiv \mu_{0}(H+M)
\end{aligned}
$$

Where $M$ is the magnetization intensity.

Substituting equation (4) into (1) and also $J=0$ in the same equation (1) we obtain

$$
\nabla \times\left(\nabla \times \frac{1}{\mu} \cdot \mu(H+M)=0\right.
$$

The equation (5) is further reduced to the generalized form of the magnetic flux continuity law

$$
\nabla \cdot \mu_{0} H=-\nabla \cdot \mu_{0} M
$$

Based on the imaginary magnetic charge created with regards to the molecular current from a pair of magnetic dipoles as done in MFL, the density of this magnetic charge can be represented as

$$
\rho_{m} \equiv-\nabla \cdot \mu_{0} M
$$

In order to represent the magnetic field intensity in terms of scalar magnetic potential $V_{m}$ the equation (8) below is obtained

$$
H=-\nabla V_{m}
$$


For which the MFL governing equation (9), representing the computational domain is obtained and satisfied

$$
\nabla^{2} V_{m}=-\rho_{m} / \mu_{0}
$$

\subsection{Formulation of the tensor based finite element model (TBFEM)}

Finite element modelling is the discretization of the computational domain into smaller fine elements called finite elements and the union of these finite elements to form a mesh [6]. The idea is to express unknown functions in terms of a linear combination of certain given functions with unknown coefficients, so that an equation (usually a matrix equation), whose entries are the unknown vectors of the unknown coefficients, are the approximate representation of the functions. In this paper, a test function is used, for which the calculation of dependent variables is done without having to directly solve the PDE. For example $\phi=S_{v i} a^{i}$ is a simple test function, as used in literature [6][7][8][9], where $\phi$ is the potential, $S_{v}$ is the shape vector, $a$ the row vector and $i=1,2,3,4 \ldots \ldots$, which is the Einstein's summation convention. According to the Einstein's summation $e_{i} f^{i}=e_{1} f^{1}+e_{2} f^{2}+e_{3} f^{3} \ldots .$. , where $e$ and $f$ are the functions of $i . S_{v}$ in the test function can further be defined as $S_{v 1}=1, S_{v 2}=x$ and $S_{v 3}=y$, whiles the values of $a^{i}$ as $a^{1}=a, a^{2}=b$ and $a^{3}=c$, where the values of $a, b$ and $c$ are unknown constants. The equation $\phi=S_{v i} a^{i}$ requires a system of $i$ equations so as to solve for the unknown constants of $a^{i}$. For this reason, 3 different potentials representing the linear variation of $\phi=S_{v i} a^{i}$ is introduced considering a triangular region of 3 nodes in space. The system of equations is then redefined as $\phi_{j}=G_{j i} a^{i}$, where $j=1,2,3$ the linear variations of nodal potentials as said earlier and $G_{j i}$ is a matrix that contains the coordinate locations of the triangle vertices. The nodal potentials $\phi_{j}$ are unknown variables, hence the constant $a^{i}$ may be defined as functions of these potentials, given by $a^{i}=g^{i j} \phi_{j}$ where $g^{i j}=G_{j i}^{-1}$ is the inverse of the matrix $G_{j i}$, which represents the geometrical weighted tensor. Combining the potentials $\phi=V_{i} a^{i}$ and $\phi_{j}=G_{j i} a^{i}$ we obtain a linear variation of the potential with the region specified by $j=1,2,3$ given as

$$
\phi=S_{v i} g^{j i} \phi_{j}
$$

Where $S_{v i} g^{j i}$ is a set of linear equations. 
2.3 Decomposition of the computational domain using tensor based finite element method (TBFEM)

The main objective of the finite element model (FEM) is to evaluate the approximate solution of a boundary value problem (BVP) that is governed by a linear partial differential equation. Using the proposed FEM model, the governing partial differential equation is solved. Partial differential equations (PDEs), are a function of continuous variables in time domain. A typical boundary value problem (BVP) can be mathematically described in a domain $\Omega \subset R_{1}, R_{2}$ or $R_{3}$ using the operator notation as shown in Eq. (11) below considering boundary conditions.

$$
L u=f
$$

Where $L$ is the partial differential operator, $u$ is the unknown function to be determined and $f$ is the given excitation function. The PDE in (9), considering boundary conditions the equation is rewritten as (11)

$$
\nabla^{2} V_{m}=-\rho_{m} / \mu_{0} \quad \text { in } \quad \Omega
$$

Where $\Omega$ is the computational domain, for which the Dirichlet, Neumann and Mixed boundary conditions can be imposed.

$$
\begin{aligned}
& V_{m}=\phi \quad \text { on } \Gamma_{D} \\
& \frac{\partial V_{m}}{\partial n}=\beta \quad \text { on } \Gamma_{N} \\
& \frac{\partial V_{m}}{\partial n}+\alpha V_{m}=\beta \quad \text { on } \Gamma_{M}
\end{aligned}
$$

Where $\phi, \beta$ and $\alpha$ are given functions and $\Gamma_{D}, \Gamma_{N}$ and $\Gamma_{M}$ represent the types of boundary conditions that can be imposed. Representing (12) above as the expression in (11), we obtained (16) below

$$
L u^{e}=f^{e}
$$

Where $u^{e}$ represents the potential $V_{m}$ for each element in the mesh and $f^{e}$ being the right hand side of the Eq. (12) also representing the excitation function for each element in the mesh. Furthermore, $u^{e}$ can be expanded, so as to express $u_{j}^{e}$ as the 
coefficient corresponding to the value of $u$ and $N_{j}^{e}$ is the weight function at the $j$-th node as shown in Eq. (17).

$$
u^{e}=\sum{ }_{j=1}^{n^{e}} u_{j}^{e} N_{j}^{e}
$$

Using the Galerkin method where $w_{i}^{e}=N_{i}^{e}$ (where the weight function is identical to the shape functions), Eq. (18) is obtained

$$
\int_{\Omega^{e}} w_{i}^{e} L u^{e} d \Omega^{e}=\int_{\Omega^{e}} w_{i}^{e} f^{e} d \Omega^{e}
$$

Now imposing the Neumann boundary conditions, the equation (18) is rewritten as (19)

$$
\int_{\Omega^{e}} w_{i}^{e} L u^{e} d \Omega^{e}=\int_{\Gamma} w_{i}^{e} L u^{e} d \Gamma
$$

Further expansion of (19) based on the gauss divergence theorem for open surfaces, the equation (20) was obtained

$$
\int_{\Gamma} w_{i}^{e} L u^{e} d \Gamma-\int_{\Omega^{e}} w_{i}^{e} L u^{e} d \Omega^{e}=\int_{\Omega^{e}} w_{i}^{e} f^{e} d \Omega^{e}
$$

Where the first expression in (20) shows the imposition of the Neumann condition over the perimeter $\Gamma$. The summation equation for this expression is further given by

$$
\int_{\Gamma} w_{i}^{e} L u^{e} d \Gamma=w^{e} L u^{e} \frac{\Gamma}{2}
$$

The second expression in Eq. (20) is then computed based on the triangular mesh formation as said in the earlier section, for which its solution is given by (22) below

$$
-\int_{\Omega^{e}} w_{i}^{e} L u^{e} d \Omega^{e}=-w^{e} L u^{e} A_{T}
$$

Where $A_{T}$ is the area of the triangular element and its obtained by computing the determinate of the matrix $\left(G_{i j}\right)$ which contains the coordinate locations of the triangular vertices. Applying the Einstein's convention as stated earlier, with the Eq. (10) using the suffix notation, the Eq. (23) is obtained 


$$
-w^{e} L u^{e} A_{T}=-A_{T} g^{l k} g^{l j} V_{m}
$$

Next, the solution of the right hand side of the Eq. (20) is obtained based on the area of the triangular element $A_{T}$ divided by the number of nodes within the triangle as shown in Eq. (24)

$$
\int_{\Omega^{e}} w_{i}^{e} f^{e} d \Omega^{e}=f^{e} \frac{A_{T}}{3}
$$

Using the Eqs. (20) - (24), the tensor based FEM for the decomposition of the governing MFL equation is obtained as shown below

$$
-A_{T} g^{l k} g^{l j} V_{m}=f^{e} \frac{A_{T}}{3}-w^{e} L u^{e} \frac{\Gamma}{2}
$$

\section{Results and discussion}

In order to verify the ability of the proposed FEM model, we modelled and simulated the distribution of magnetic field between parallel conductors placed in the XY plane. The field distribution was then computed on the XY direction, with the $Z$ component kept constant $(Z=0)$. The results of this implementation can be seen in the Figure.2 $(a-b)$ below.

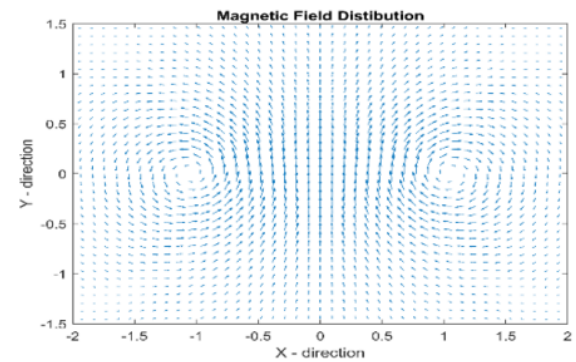

(a)

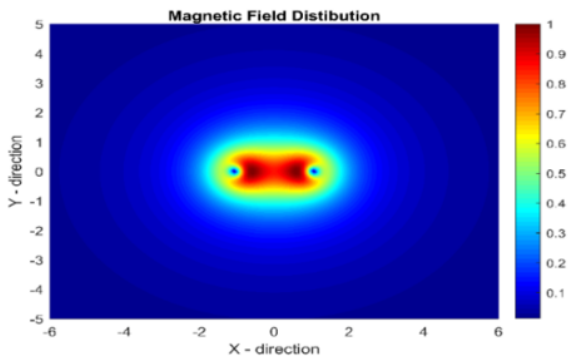

(b)

Figure. 2 (a) and (b) Magnetic field distribution between parallel conductors (permanent yokes) simulated using TBFEM implemented in MATLAB

From the Figure. $2(a-b)$, the uniformity in the magnetic field distribution between two yokes can be evidently seen, for which the proposed FEM can be verified to yield results that agrees with results from simulations using the traditional FEM models.

Next, we calculated the magnetization of specimen, according to the magnetic field intensity, for which the leakage field is also obtained. Here, the variables for magnetizing the defect material is chosen based on B-H characteristics of a material [10]. The choice of material (low carbon fiber) was based on the common practice in MFL. More so, the magnetization variables were computed, with the traditional FEM model as a standard reference, so as to ensure standard accuracy in MFL signals when material saturation is 
achieved. Furthermore, this implementation is done to prove the validity of the proposed model in yielding results, that are consistent with results obtained from traditional FEM in similar conditions. The material size is set to $0.1,0.5$ and $0.006 \mathrm{~m}$ respectively in the $\mathrm{x}$, $\mathrm{y}$ and $\mathrm{z}$ directions, while the defect size is set to Length $(\mathrm{L}=2 \mathrm{~mm})$, Width $(\mathrm{W}=1 \mathrm{~mm})$ and Depth $(\mathrm{D}=3 \mathrm{~mm})$. Using the both the traditional FEM and the TBFEM, simulation is done. Comparing the Y-components of magnetic field intensity at a liftoff position of 1- 4mm, the results are shown in Figure. 3 (a-d) below, it can be seen that the field distribution calculated by both the traditional FEM and the proposed FEM (TBFEM) greatly agree except for a minimal error of about $0.54 \%$. However, it is also observed that when lift-off is $1 \mathrm{~mm}$, there exist a marginable amount of error in peak distribution between the two models. This is due to the fact that when lift-off is relatively small, marginal testing errors can be realized and when liftoff is excessively huge, the peak distribution completely vanishes, resulting to a complete erroneous testing. Therefore, as shown in the Figure. 3 (a-d), varying lift-off from $1-4 \mathrm{~mm}$ is used, so as to verify the effect of lift-off on leakage field signal and to recommend or validate lift-off parameters for best result. Based on the simulation results as shown in Figure 3 (a-d), we can assume that, a standard lift-off of $3-4 \mathrm{~mm}$ is suitable for achieving best results. As anything above this will or might result to erroneous test. Finally comparing the computation speed between the two models, the traditional FEM uses about 304s for the computation of the above data using a windows based computer of $2.4 \mathrm{ghz}$ speed, 8GB RAM, whiles the TBFEM uses about $24 \mathrm{~s}$ for the same data with the same computer specification. Hence the purpose of the TBFEM is achieved through the reduction of matrixes.

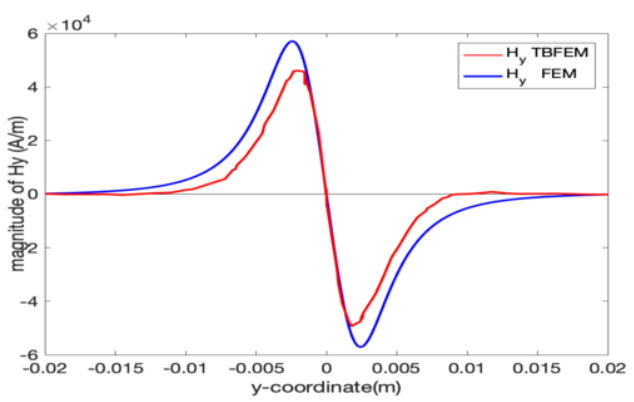

(a)

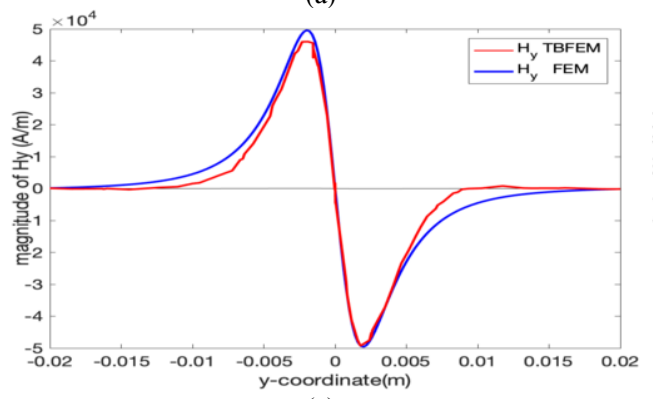

(c)

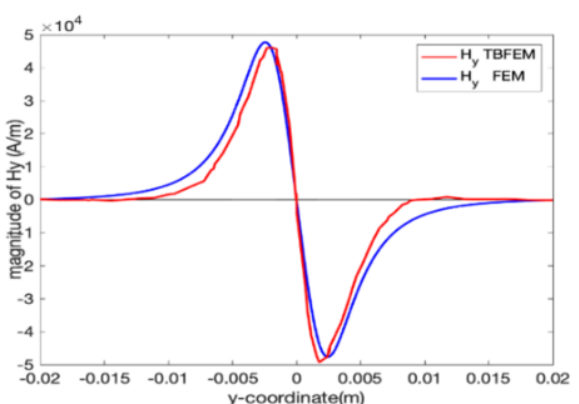

(b)

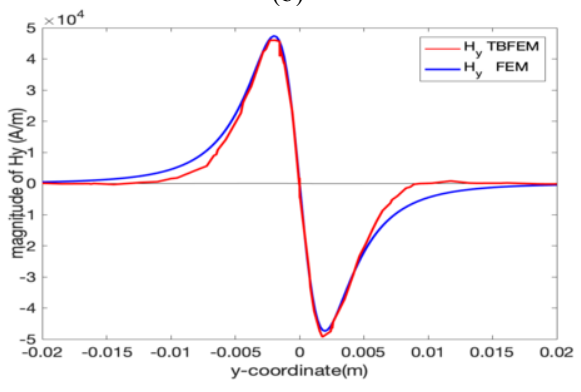

(d)

Figure. 3 Magnitude magnetic field intensity (a) when liftoff $1 \mathrm{~mm}$ (b) when liftoff is $2 \mathrm{~mm}$ (c) when liftoff is $3 \mathrm{~mm}$ (d) when liftoff is $4 \mathrm{~mm}$ respectively 


\section{Conclusions}

This paper presents a tensor based finite element model for the calculation of leakage fields in MFL. This model eliminates the bulky nature of traditional FEM based on matrix formation by reducing the set of matrixes into a single Equation without changing the original meaning. With the proposed method, finer meshes can be generated and calculated within a short period of time with minimum computer resources.

\section{References}

[1] V. Suresh and A. Abudhahir, "An Analytical Model for Prediction of Magnetic Flux Leakage from Surface Defects in Ferromagnetic Tubes," Meas. Sci. Rev., vol. 16, no. 1, pp. 8-13, 2016.

[2] S. M. Dutta, F. H. Ghorbel, and R. K. Stanley, "Dipole Modeling of Magnetic Flux Leakage," vol. 45, no. 4, pp. 1959-1965, 2009.

[3] J. H. Hwang, W. Lord, and M. Ieee, "Finite Element Analysis of the Magnetic Field Distribution Inside a Rotating," no. 5, 1974.

[4] S. Nagu, "Finite Element Modeling of Magnetic Flux Leakage Technique in Plates with Defect and without Defect," Int. J. Mod. Eng. Res., vol. 3, pp. 3452-3455, 2013.

[5] Comsol and S. M. FORSCHUNG, "Simulation of Magnetic Flux Leakage Inspection,” pp. 8-9.

[6] Ö. Özgün and M. Kuzuoğlu, MATLAB-based Finite Element programing in Electromagnetic Modeling, 1st Editio. CRC Press Taylor \& Francis, 2019.

[7] Altair university, Introduction to Nonlinear Finite Element Analysis using Optistruct, 2nd Editio. Michigan, USA,: Altair HyperWorks Documentation Team, 2018.

[8] C. Hernandez and M. A. Arjona, "Application of tensor analysis to the finite element method," Appl. Math. Comput., vol. 219, no. 9, pp. 4625-4636, 2013.

[9] R. A. Arciniega and J. N. Reddy, "Tensor-based finite element formulation for geometrically nonlinear analysis of shell structures," Computer Methods in Applied Mechanics and Engineering, vol. 196, no. 4-6. pp. 1048-1073, 2007.

[10] Y. Shi, C. Zhang, R. Li, M. Cai, and G. Jia, "Theory and application of magnetic flux leakage pipeline detection," Sensors (Switzerland), vol. 15, no. 12, pp. 31036-31055, 2015. 